\title{
Enhanced Reactive Location Service for Packet Delivery in Vehicular Ad-Hoc Networks
}

\author{
${ }^{1}$ Mrs. Sree Divya. Nadella ${ }^{2}$ Mr. Ramesh. Vatambeti ${ }^{3}$ Mr. Veeramallu. Bobba \\ ${ }^{1}$ Research Scholar, KL University, Vijayawada, AP, India. \\ E-Mail: sridivya1234@gmail.com \\ ${ }^{2}$ Professor, IT, Mahatma Gandhi Institute of Technology, Hyderabad, India. \\ E-Mail: ramesh.v@mgit.ac.in \\ ${ }^{3}$ Professor, CSE, Amritasai Institute of Science \& Technology, Vijayawada, India.
}

\begin{abstract}
VANETs are special kind of Mobile Ad Hoc Networks (MANETs) that are formed between moving vehicles on an as-needed basis. Routing in VANET uses a greedy routing protocol which requires geographical information like location of any vehicle.

The primary objective of this paper is to reduce the transmission delay by using some Road Side Units (RSU) already deployed along the roads. RSU gather information about all the vehicles which are connected in its range. Here each and every vehicle need not be communicated but the vehicles that are nearest and which have the information about location of destination are only involved. We use enhanced RLS which is hybrid communication from source to destination. It results to reduce the flooding requests and make communication faster which leads to improve the packet delivery ratio and reduce the transmission delay or end to end delay. In addition to this the source vehicle sends a request with its velocity to RSU, it will communicate with other RSU's for the destination vehicle. After finding the destination it will send the request with velocity of the destination. Based on the velocity of destination and intermediate nodes the source will increase its velocity to avoid the frequent link breakage in order to reduce the transmission delay. The proposed algorithm is simulated using NS2 and results shows that ERLS decreases the transmission delay and increases the packet delivery ratio.
\end{abstract}

Keywords - VANET, Location-based Services, Geographic Routing, Hybrid Architecture.

\section{Introduction.}

VANET belongs to wireless communication network in which communication between vehicles takes place. The vehicles act as nodes in the network which form a network without any additional infrastructure. With rapid growing number of vehicles, saturation of the transportation infrastructure, and traffic congestion, accidents and transportation delays become unavoidable. Several mechanisms are implemented to improve the transportation systems as well as developed better and efficient routing mechanisms. VANETS have many applications in Road Safety, Passenger convenience etc. VANET communication has recently become an increasingly popular research topic in the area of wireless networking. A VANET turns every participating vehicle into a wireless router or node allowing vehicles to connect and create a network. Routing in VANET's is still a challenging issue due to its characteristics like high mobility of nodes, frequent disconnections, highly partitioning network. When the vehicle density is low, VANETs have a higher probability of getting disconnected frequently.

VANET will become world's largest ad-hoc network, communication between vehicles to vehicle is in different types as Vehicle to Vehicle (V2V) - Vehicle to Vehicle communication is suitable for short range vehicular network. It provides real time safety, fast and reliable. It does not need any roadside infrastructure. It is not very useful in case of sparsely connected network or low density vehicular network. In V2V warning messages are broadcasted from vehicle to vehicle. Vehicle to Roadside (V2R) -Vehicle to Roadside provides communication between vehicles and the roadside units. It makes use of pre-existing network infrastructure such as wireless access points. In V2R warning messages are sent to roadside units and then from that roadside units warning messages sent to the vehicles. Hybrid architecture communication this combines both Vehicle to Vehicle ad-hoc networks (VtoV) and Vehicle to Roadside network architecture (V2R).

The Routing protocols of VANET's must require the Geographical information to find about source location and destination location. Source location can be given by different devices like GPS and the destination location can be found by using location services like HLS, RLS. The location service had an advantage of deployed RSU's on roads in which each RSU has information about all the vehicles under its range and can also exchange the position of nodes. In this paper instead of communication between the vehicles, we can communicate between vehicle to RSU and RSU's to other RSU's. With this we have an advantage that the source node will not send request to each and every node it directly sends to RSU's, by this we can reduce the load on the network. In addition to this the RSU's can also have an ability to give the information about relative velocity of 
vehicles moving in its range. The source can receive the velocity of the all intermediate nodes and destination by HLS. With the information of relative velocity of the intermediate nodes it can reduce the link breakage.

\section{Related Work}

Boubakeur Moussaoui[1], proposed an improved location service(iRLS) an extension of the RLS service. iRLS takes profit of a wireless backbone based on RSUs to catch the destination's position. This allows reducing the overhead instead of flooding requests and also makes the communication faster, the closest RSU will reply with the actual location.

Neha [2], Analysis of Hybrid GPSR and Location Based Routing Protocol in VANET, presented various routing protocols and out of all Position based routing protocols is found to be suitable for Vanet. GPSR is one of the most suitable position based routing protocol. This presents a hybrid approach PHRHLS (A Movement Prediction based Joint Routing and Hierarchal Location Based Service) coupling GPSR protocol and HLS location service with mobility algorithm.

K.Prasanth,[3] in his paper argues the use of information on vehicles' movement information (e.g., position, direction, speed of vehicles) to predict a possible link-breakage event prior to its occurrence. In his proposal a Reliable Directional Greedy routing (RDGR), a reliable position based routing approach which obtains position, speed and direction of its neighboring nodes from GPS. This approach incorporates potential score based strategy, which calculates link stability between neighbor nodes in distributed fashion for reliable forwarding of data packet.

Young-Bae Ko and Nitin H. Vaidya[4] suggests an approach to utilize location information (for instance, obtained using the global positioning system) to improve performance of routing protocols for ad hoc networks. By using location information, the proposed Location-Aided Routing (LAR) protocols limit the search for a new route to a smaller "request zone" of the ad hoc network. This paper describes how location information may be used to re-duce the routing overhead in ad hoc networks. We present two location-aided routing (LAR) protocols. These protocols limit the search for a route to the so-called request zone, determined based on the expected location of the destination node at the time of route discovery. Simulation results indicate that using location information results in significantly lower routing overhead, as compared to an algorithm that does not use location information.

Location Based service is flooding based and can be composed of reactive and proactive services. In proactive service every node floods its geographic information through all the network periodically. Thus, all the nodes are able to update their location tables. the network by location update messages, several techniques to reduce the congestion were used. One of them is to tune the update frequency with the node mobility (the more node is moving fast, the higher update location frequency is used).So the update frequency decreases with the distance to the node. The reactive service is a node with high mobility sends more update location packets which lead to less packets than a simple flooding scheme without affecting the network performances. The location response is sent when receiving a location request. This avoids the overhead of useless location information of some nodes updated and never used. But, it adds high latencies not suitable in VANETs. One of these known services is Reactive Location Service (RLS). Thus, the location-based service consists of two components: location update and Location Request.

The geographic routing are suitable for large scale dynamic networks. The first routing protocol using the geographic information is the Location-Aided Routing (LAR) [4]. This protocol used the geographic information in the route discovery. This latter is initiated in a Request Zone. If the request doesn't succeed, it initiates another request with a larger Request Zone and the decision is made on a routing table. The first real geographic routing protocol is the Greedy Perimeter Stateless Routing (GPSR) [10]. It is a reactive protocol which forwards the packet to the target's nearest neighbor (Greedy Forwarding approach) until reaching the destination. Therefore, it scales better than the topology-based protocols, but it does still not consider the urban streets topology and the existence of obstacles to radio transmissions. Another geographic routing protocol is the Geographic Source Routing (GSR) [11]. It combines geographical information and urban topology (street awareness). The sender calculates the shorter path (using Djikstra algorithm) to the destination from a map location information. Then, it selects a sequence of intersections (anchor-based) by which the data packet has to travel, thus forming the shortest path routing. To send messages from one intersection to another, it uses the greedy forwarding approach. The choice of intersections is fixed and does not consider the spatial and temporal traffic variations. Therefore, it increases the risk of choosing streets where the connectivity is not guaranteed and losing packets. 


\section{Proposed Approach: Enhanced Reactive Location Service for VANET}

Suppose if vehicle V5 want to communicate and send data to destination V8, if the destination is in its range it directly sends to the destination, otherwise it broadcast a request to find a suitable route by sending a query to nearest RSU1, if the destination is in its range it will send the response with velocity, position, route of destination by using DGRP (Directional Greedy Routing Protocol). If the destination is not it send the request to other neighbor RSU's to find the destination by using the hybrid architecture. After receiving the response from RSU 1, V5 starts sending data using the greedy forwarding approach. In the Fig .1 , RSU 1 sends to RSU 3 and it communicate with V9 .Here it send to RSU-3 to RSU 4 which and communicate with V7 which is nearer to destination V8. Once the destination is found it sends its position, velocity to the source and sends data from V5 to V9, V7 and finally to V8. Therefore by using the hybrid architecture destination's location could be found more rapidly than using V2V communications when a destination node is far away from the source node.

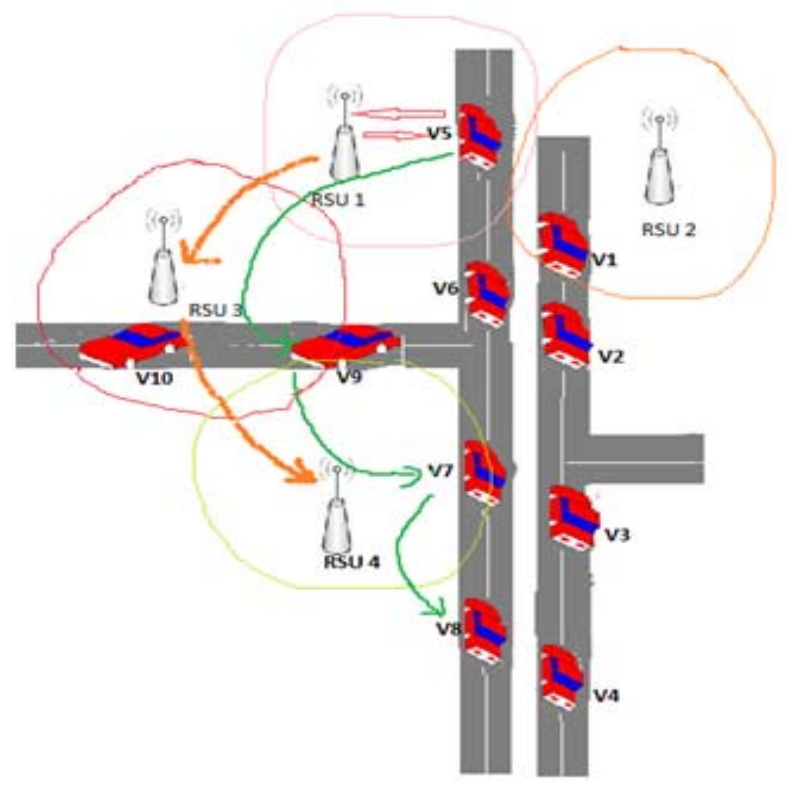

Fig.1. An Example Scenario.

In the given algorithm, Enhanced Reactive Location service the source will broadcast the request to find position of destination with its velocity, the nearest RSU will give response if it's in range, otherwise It will communicate with other RSU' $s$ to find position of destination and relative speed of intermediate nodes on direct path from source to destination by using DGRP, then it will send the response to the source, source will check the velocity of destination and intermediate nodes if its velocity is less than the destination and intermediate nodes it will increase its velocity equal to intermediate node velocity or it selects the nodes which are equal to relative speed of the source node, with this the link breakage will be reduced and flooding to every node in the RSU's will be reduced i.e. the communication from RSU to RSU will increase the network performance and reduce the end to end delay.

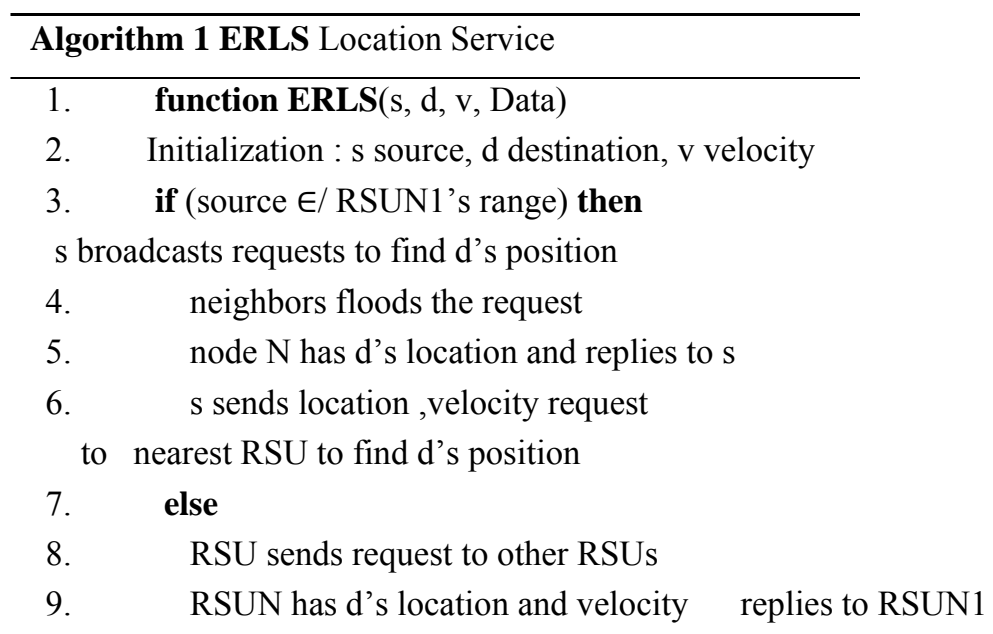


10. RSUN1 forwards response to $\mathrm{s}$

11. end if

12. next $\leftarrow \mathrm{s}$

13. while (next !=d) do

14. choose node $x$ with relative velocity of next hop using greedy approach

15. if $(\mathrm{v}(\mathrm{s})<\mathrm{v}(\mathrm{x}) \& \& \mathrm{v}(\mathrm{d}))$ then

16. $\quad \mathrm{v}(\mathrm{s})=\mathrm{v}(\mathrm{x})$

17. $\mathrm{s}$ sends data to $\mathrm{x}$

18. next $\leftarrow \mathrm{x}$

19. end while

20. Data reaches destination d

\section{1.end function}

\section{Simulation Results:}

The simulator used was Ns-2.The geographic routing protocol used is GPRS[10].the GPRS routing protocol was used with certain area. Table.1 gives some simulation parameters. Our experiment results in Fig 2. The network behavior with RLS and the behavior of our proposed method shows the delay to send a message depending on the network from 10 to 100 nodes is less for ERLS than RLS . In same way Fig 3. Shows a higher PDR of ERLS than the RLS.

Table.I.Simulation Parameters

\begin{tabular}{|c|c|}
\hline Parameter & Value \\
\hline Channel type & Channel/WirelessChannel \\
\hline Network interface & Phy/WirelessPhyExt \\
\hline MAC layer & $802.11 \mathrm{p}$ \\
\hline Interface queue length & GPSR \\
\hline Ad-hoc routing protocol & RLS \\
\hline Location-based service & $4,8,12,16$, and $22 \mathrm{~s}$ \\
\hline Location cache maximum age & $2 \times 2$ KM ${ }^{2}$ \\
\hline Area & $0,5 \mathrm{~s}$ \\
\hline Number of nodes & $4 \times 100$ packets $/$ node \\
\hline GPRS beacon interval & $12 \mathrm{~KB}$ \\
\hline CBR traffic & $1 \mathrm{~s}$ \\
\hline CBR packet size & \\
\hline CBR sent interval & \\
\hline
\end{tabular}

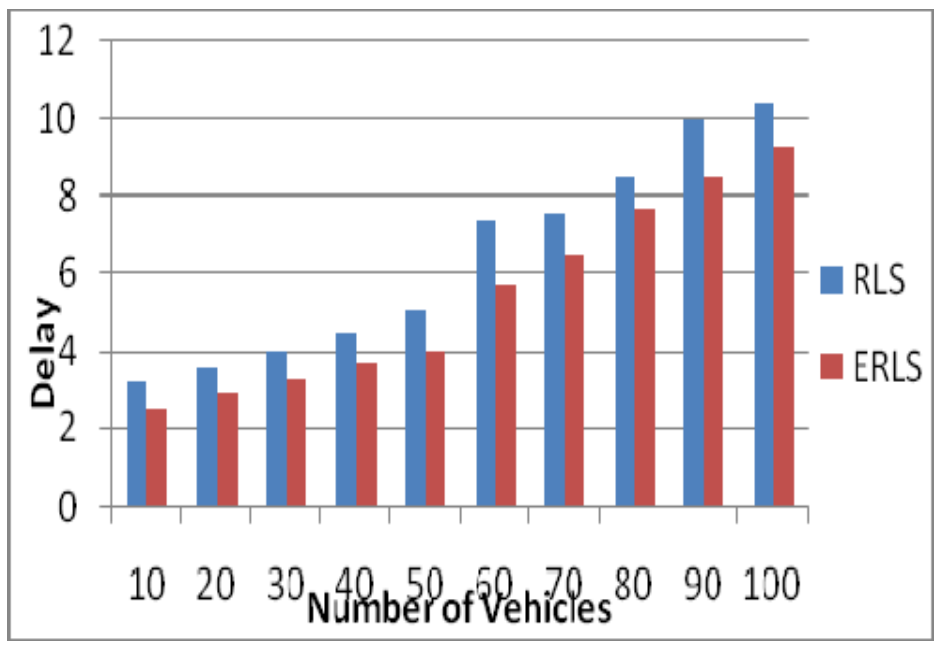

Fig 2. Average Packet Transmission delay 
In Fig. 2. It shows the decrease in transmission delay by using the ERLS than RLS.

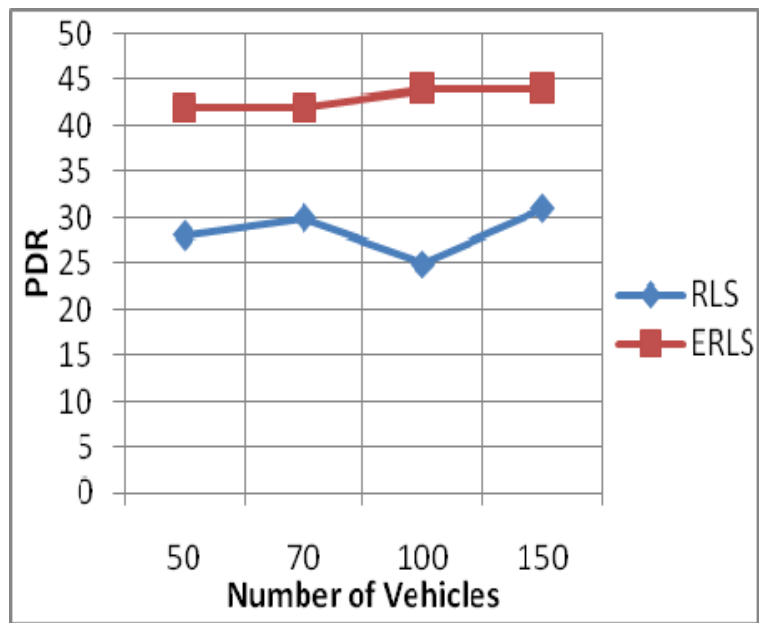

Fig.3. Impact of the number of vehicles on the achieved PDR average

The results show that our protocol is more than $15 \%$ increase in PDR by use of ERLS. This observed result is quite predictable since the RLS process uses the V2V communications (a RSU is considered as a vehicle also) then the request is sent through the whole network without any efficiency. Difference to RLS, ERLS takes advantage of the RSU backbone which provide a fast connection between the initial RSU and the closest to the destination.

\section{Conclusion \& Future Work:}

This paper presents ERLS by this location service we can communicate vehicle to RSU and RSU to RSU which are deployed on roads. RSU's have the information about the vehicles and they can also exchange data of the nodes position and velocity. This improves certain parameters like network performance, transmission delay of packets, packet delivery ratio and end to end delay, also it reduces the link breakage. As a future work, it can be extend with use of RSU's we also use soft computing techniques in other king of protocols.

\section{References:}

[1] Boubakeur Moussaoui, Hacène Fouchal, Marwane Ayaida and Salah Mermiz, "Unicast Routing on VANETs" ,IEEE , Federated Conference on Computer Science and Information Systems pp. 1089-1092,2016.

[2] Neha, Isha, "Analysis of Hybrid GPSR and Location Based Routing Protocol in VANET" (IJCSIT) International Journal of Computer Science and Information Technologies, Vol. 6 (1), 2015, 674-677.

[3] K. Prasanth, D. K. Duraiswamy, K. Jayasudha and D. C. Chandrasekar, "Improved packet forwarding approach in Vehicular ad hoc networks using RDGR algorithm", arXiv preprint arXiv:1003.5437, 2010.

[4] Y.-B. Ko and N. H. Vaidya, "Location-aided routing (LAR) in mobile ad hoc networks," Wireless Networks, vol. 6, no. 4, pp. 307321, Jul. 2000.

[5] R. Rana, S. Rana and K. C. Purohit, "A review of various routing protocols in VANET," International Journal of Computer Applications, vol. 96-18, 2014.

[6] C. J. Huang, Y. T. Chuang, . Y. J. Chen, D. X. Yang and I.F. Chen, "QOSaware roadside base station assisted routing in vehicular networks." Engineering Applications of Artificial Intelligence, 22(8), 1292-1301. 2014.

[7] G. J. Borsetti, "Infrastructure-assisted geo-routing for cooperative vehic-ular networks," IEEE Vehicular networking conference (VNC), 2010, pp. 255-262.

[8] Y. Peng, Z. Abichar and J.M. Chang, "Roadside-aided routing (RAR) in vehicular networks." IEEE International Conference on ICC'06.

[9] Käsemann, M., Füßler, H., Hartenstein, H., Mauve, M. (2002). A reactive location service for mobile ad hoc networks*. Universität Mannheim/Institut für Informatik, Technical report REIHE INFOR-MATIK 14/2002. 14/2002.

[10] B. Karp and H. T. Kung, "GPSR: Greedy Perimeter Stateless Routing for Wireless Networks," in Proceedings of the 6th annual international conference on Mobile computing and networking (MobiCom'00), New York, NY, USA, 2000, pp. 243-254.

[11] C. Lochert, H. Hartenstein, J. Tian, H. Fuessler, D. Hermann, and M. Mauve, "A routing strategy for vehicular ad hoc networks in city environments," In Proceedings of the IEEE Intelligent Vehicles Symposium, 2003, pp. 156-161.

[12] Xianghui Cao, Lu Liu, Yu ChengLin,X. Cai, Changyin Sun(2016). "On Optimal Device-to-Device Resource Allocation for Minimizing End-to-End Delay in VANETs",IEEE Transactions On Vehicular Technology, Vol. 65, No. 10, pp. 7905- 7916.

[13] S. Nallusamy, S. Appavupillai, and S. Ponnusamy, "Mobile Agents based Reliable and Energy Efficient Routing Protocol for MANET", International Journal of Intelligent Engineering and Systems, Vol.9, No.3, pp. 110-116, 2016.

[14] Avinash P. Ingle, "Hybrid routing protocol in Vehicular ad-hoc network foar Highly Heterogeneous traffic Environment", International Journal of Computer Science \& Engineering Technology, Vol. 7 No. 03, pp. 55-59, 2016.

[15] Benslimane, Abderrahim, Tarik Taleb, and Rajarajan Sivaraj,'Dynamic clustering-based adaptive mobile gateway management in integrated VANET-3G Heterogeneous Wireless Networks', Selected Areas in Communications, vol. 29, Issue 3, pp. 559-570, 2011.

[16] AL-Hashimi, Haider Noori, Kamalrulnizam Abu Bakar, and Kayhan Zrar Ghafoor, 'Inter-domain proxy mobile ipv6 based vehicular network', Network Protocols and Algorithms, vol.2, issue 4, pp. 1-15, 2011. 


\section{About the Authors:}

Mrs. N. Sree Divya, Research Scholar, KL University and working as Assistant Professor in the Department of IT at Mahatma Gandhi Institute of Technology, Hyderabad. She has completed both B.Tech and M.Tech from JNTU Hyderabad. She has 10 years of teaching experience. She has published 8 research papers in Journals and Conference proceedings. Her research interests include Mobile Ad-hoc Networks and Data base systems.

Dr. V. Ramesh, Professor in the Department of Information Technology, obtained B.Tech in CSE from S.V. University, M.Tech and PhD in Computer Science and Engineering from Sathyabama University, Chennai. He has 13 years of teaching experience. He has authored two books "Principles of Operating Systems" by University Science Press, New Delhi. \& “ Preemptive DSR for Mobile Ad Hoc Networks” by Lambert Lap Publishers, Germany. He has published 52 research papers in various refereed International Journals and Conference Proceedings. His research interests include Mobile Ad-hoc Networks and Data Mining.

Dr .B.Veeramallu working as Professor \& Head of the Department in Amritasai Institute of Science \& Technology in the department of CSE. He completed B.E in computer science and Engg from Madras University, M.Tech in Computer Science and Engineering from JNTUH and PhD in Computer Science and Engg from K.L.University. He has 16 papers published in journals. He has 17 years of experience in teaching from various organizations. 